\title{
Can Vocabulary Size Predict Narrative Abilities in Children with SLI?
}

\author{
Maria Kambanaros ${ }^{1}$, Kleanthes K. Grohmann², Eleni Theodorou ${ }^{2}$ \\ and Michalis Michaelides ${ }^{2}$ \\ ${ }^{1}$ Cyprus University of Technology, ${ }^{2}$ University of Cyprus \\ kambanaros@gmail.com, kleanthi@ucy.ac.cy, \\ theodorou.d.eleni@ucy.ac.cy,michaelides.michalis@ucy.ac.cy
}

\begin{abstract}
Correlating expressive vocabulary skills with narrative microstructure measures such as morphosyntactic skills revealed that both are powerful tools to assess language-impaired children. However, for expressive vocabulary to have a predictive value on narrative performance, younger children with SLI should be investigated. Our results lend support to a unified model of SLI (Rice 2003) revealing that both expressive vocabulary and narrative re-tell microstructure are delayed in school-aged Greek Cypriot children with SLI compared to their typically developing peers.
\end{abstract}

\section{Introduction}

The larger research agenda pursued by the Cyprus Acquisition Team is to investigate typically and atypically language-developing children in Cyprus from mono- but also bi- and multilingual backgrounds. Our research so far has shown, among other things, that children with specific language impairment (SLI) have (i) smaller vocabularies, especially in relation to common, concrete object and action words (Kambanaros \& Grohmann 2010), and (ii) difficulties producing oral narratives (Theodorou \& Grohmann 2010, Theodorou 2012) compared to age-matched peers with typical language development (TLD). Taken together, then, narratives and vocabulary and/ or naming abilities do separately serve as diagnostic indexes for SLI. The present study aims to explore vocabulary size as a possible predictor of narrative ability in two groups of children, those with TLD and children with SLI.

The study was carried out in the Republic of Cyprus and "Greek at large" is the language under investigation. As native acquirers of Cypriot Greek, the local variety of the language, Greek Cypriot children are not exposed to Standard Modern Greek before school entry, at least not within typical everyday life (other

\section{(cc) BY-NC-ND}


than cartoons on television, for example). Cypriot Greek differs from the standard variety in substantial ways, which do not play a role here (but see e.g. Grohmann et al. 2006, Grohmann 2011, Grohmann \& Leivada 2012, and Grohmann et al. 2012). The linguistic situation of (Greek-speaking) Cyprus is described as diglossia, where Standard Modern Greek enjoys sociolinguistically "high" status, while Cypriot Greek is the "low" variety. For the purposes of the present study, we did not look for differences between the two varieties but counted all Greek responses equally well regardless of their origin (whether lexical, phonological, or morphosyntactic). As such, we refer to the language under investigation as "Greek" throughout.

\section{Background}

The description of language disorders in SLI is usually based on (i) the characteristics of children's spontaneous speech output and (ii) children's performances on specific linguistic tasks tapping into the different language components under investigation (such as syntax, semantics, phonology, and morphology, but also pragmatics as well as lexicon and word finding).

\subsection{Narrative and SLI}

Many cross-linguistic studies have shown that children with SLI are less skilled both in composing and in producing oral narratives compared to age-matched peers with typical language development. In fact, the narratives of children with SLI are described as less competent and more immature with respect to length, lexical diversity, sentence complexity, and content (Gillam \& Pearson 2004). Furthermore, difficulties with narratives is considered an area of spoken language in which delay is less likely to resolve over time for children with SLI (Manhardt \& Rescorla 2002).

Concerning the analysis of narratives, researchers typically differentiate between a micro- and a macrostructural analysis. Microstructure incorporates the semantic and syntactic complexity of narratives, using measures such as mean length of utterance, number of clauses per T-unit, quantity of vocabulary, and diversity of vocabulary. Macrostructure refers to the general, global characteristics of a narrative focusing on coherence and organization of the narrative.

\subsection{Vocabulary and SLI}

Vocabulary development in children with SLI has been examined both for a general delay of vocabulary overall and for specific delays within different 
word classes, such as a particular difficulty with acquiring verbs compared to typically language-developing peers (see Rice 2003 on a unified model of SLI). A number of investigations (mainly for the English language) have shown that difficulties with word knowledge including storing, organizing and/or accessing lexical information is prominent in SLI. Several explanations have been put forward including the storage hypothesis (Leonard 1998) which suggests that children with SLI are slower to learn new words, have less elaborate semantic representations, and fewer lexical connections with words within their lexicon. Alternatively, the retrieval hypothesis (Newman \& German 2002) posits that children with SLI have difficulties with lexical access because of inefficient word retrieval mechanisms and the difficulties do not lie within lexical entries or their connections.

\section{Methodology}

\subsection{Participants}

Two groups of children participated in this study: 11 children with TLD (aged between 4;6-6;11 years;months) and 10 children diagnosed with SLI (aged between 5;2-6;10). Participant demographics are presented in Table 1.

Table 1.

Child demographics according to group.

\begin{tabular}{|l|l|l|}
\hline & TLD $(n=11)$ & SLI $(n=10)$ \\
\hline Boys (number of participants) & 5 & 8 \\
\hline Girls number of participants) & 6 & 2 \\
\hline Mean age (in months) & 70 & 71 \\
\hline Range of ages (in months) & $54-83$ & $62-82$ \\
\hline Standard Deviation & 8.02 & 7.18 \\
\hline
\end{tabular}

The children with TLD were recruited randomly from public pre-primary schools in Limassol after approval from the Ministry of Education and upon written parental consent. Children with SLI were recruited from speech and language therapists working in public schools and/or private practice in Limassol. No participating child presented with evidence of hearing loss, neurological impairment, gross motor or articulation difficulties, social, emotional or behavioral problems. 
All children with language impairment were receiving speech and language therapy and were diagnosed with SLI by their speech and language therapists, prior to the study. A large testing battery developed to identify SLI in Cypriot Greek-speaking children (Theodorou 2013) was used in order to assess and confirm the earlier diagnosis of the participants of this study. These measures included the Raven's Coloured Progressive Matrices (RCPM; Raven et al. 2000), which assesses non-verbal performance; the Diagnostic Verbal IO Test (DVIO; Tsimpli \& Stavrakaki 2000), which consists of five subparts that test vocabulary (naming), comprehension and production of morphosyntax, comprehension of metalinguistic concepts, and sentence repetition; the Peabody Peabody Picture Vocabulary Test (PPVT; Dunn \& Dunn 1981), which assesses children's receptive vocabulary abilities; and the Word Finding Test (WFT; Vogindroukas et al. 2009), which assesses the extent to which pictures of objects can be named correctly.

The participants' mean scores are shown in Table 2.

Table 2.

Mean scores (standard deviations) on the testing battery for SLI diagnosis.

\begin{tabular}{|c|c|c|c|c|}
\hline Measures & Group & \multicolumn{2}{|l|}{ TLD } & SLI \\
\hline \multicolumn{2}{|l|}{ RCPM } & & 85.4 (standard) \\
\hline \multicolumn{2}{|c|}{ DVIO - Production of Morphosyntax } & \multicolumn{2}{|l|}{$19.9(2.11)$} & $12.3(2.09)$ \\
\hline \multicolumn{2}{|c|}{ DVIO - Comprehension of Morphosyntax } & \multicolumn{2}{|l|}{$26.4(2.46)$} & $22.4(1.84)$ \\
\hline \multicolumn{2}{|c|}{ DVIO-Sentence Repetition } & \multicolumn{2}{|l|}{$46.8(1.8)$} & $40.8(2.70)$ \\
\hline \multicolumn{2}{|c|}{ DVIO - Vocabulary Naming } & \multicolumn{2}{|l|}{$22.3(1.58)$} & $15.7(2.2)$ \\
\hline \multicolumn{2}{|c|}{$\begin{array}{l}\text { DVIO-Comprehension of Metalinguistic } \\
\text { Concepts }\end{array}$} & \multicolumn{2}{|c|}{$20.1(2.45)$} & $17.5(1.29)$ \\
\hline \multicolumn{2}{|l|}{ PPVT } & \multicolumn{2}{|c|}{$79.3(32.02)$} & $69.3(16.63)$ \\
\hline \multicolumn{2}{|l|}{ WFT } & $26.5-33.2$ & 30.2 (SD 7.9) & $27.5(3.83)$ \\
\hline
\end{tabular}

\subsection{Materials}

In the present study, two tasks were correlated in order to investigate whether the vocabulary size of children with SLI can predict their narrative abilities: (i) vocabulary scores on two confrontation naming tasks, the Cypriot Object and Action Test (COAT: Kambanaros et al. 2013) and the vocabulary subtest of the DVIO, and (ii) a narrative re-tell task using the Bus Story Test (BST: Renfrew 1969 [1997]). All tasks are described below. 


\subsubsection{Vocabulary Measures}

The COAT, consisting of seventy-four coloured photographs measuring $10 \times 14 \mathrm{~cm}$, was administered to evaluate lexical retrieval of object (35 pictures) and action names (39 pictures). Object names were single, concrete inanimate nouns and included manipulated instruments such as garage tools, garden equipment, kitchen utensils, household items, and office and personal implements used for activities of daily living. Object names were not controlled for gender: 5 nouns were masculine, 14 feminine, and 16 neuter. All selected verbs were monotransitive pictureable actions with either simple internal word structures of [root + affix] or more complex ones of [root + affix + affix]. Actions were restricted to (perhaps outdated) stereotypical roles, that is, a woman is shown performing household activities (e.g. sweeping), and a man is performing more manly duties (e.g. hammering). All action names corresponded either to an instrumental verb (where an instrument is part of the action, e.g. cutting) or to a non-instrumental verb (e.g. climbing).

The vocabulary subtest of the DVIO consists of 27 black and white drawings: 13 drawings representing common nouns that could be divided into high (e.g., book, car hat, spoon) and low frequency items (e.g., violin, bridge, binoculars, thermometer), and 14 drawings representing verb phrases (e.g., pushed the boy).

\subsubsection{The Bus Story}

The BST is a screening test of verbal expression which examines story retelling with picture support. It can demonstrate difficulties with verbal expression as well as phonological, semantic, grammatical, and sequencing problems. The test consists of a wordless picture book which describes the story of a naughty red bus. It is a multi-episode story. The scenario is designed to motivate the need to identify different characters and to express temporal and causal relationships between events and between characters.

\subsection{Procedures}

The COAT, the DVIQ vocabulary subtest, and the BST were presented in one or two sessions and in random order. All children were tested in their schools, in speech and language clinics, or in their houses in a quiet room. Testing was conducted individually. The procedure for the administration of each test follows.

For the COAT, children were asked to name the object or action represented in the photograph in a single word (one-word target response). The stimulus question for the action pictures was: "Tell me in one word, what is he/she doing?". Action names were required in the third person singular. The stimulus 
question for the object pictures was: "Tell me in one word, what is this?". The stimulus question was repeated once if needed. If no response was given, the item was scored as incorrect. No time limits were placed and self-correction was allowed. Responses were recorded and transcribed verbatim. For the purposes of this study errors were scored either as correct or incorrect.

For the DVIQ vocabulary subtest, the "What's this?" question was used to elicit naming of the nouns and foe the verbs the question incorporated the subject of the verb phrase (e.g., "What's the girl/child/man/woman/grandmother doing?").

For the BST, the experimenter told each child the short story about a red bus, while the child was looking through the picture book illustrating the story. Then the experimenter asked the child to retell the story as close to the original as possible. The child then retold it, using the pictures as prompts. During the retellings, no reinforcement was provided other than encouragement with head nods and fillers.

All narrations were audio-recorded and transcribed using the IPA (International Phonetic Association 1999). Utterances were then divided into sentences and evaluated with respect to five measures, three that the BST suggests and two additional ones: the amount of original information included (Information), the number of subordinate clauses (Subordinates), the mean sentence length of the longest five sentences (A5LS), the mean length of utterances based on words (MLU-word), and the total amount of sentences used (Sentences). All but the first of these are based on microstructural analysis.

The detailed scoring scheme used is the following:

A. Information: The semantic complexity is measured using a norm-referenced information score that the BST provides. The number of relevant information included in the story are indicated following Renfrew's (1969 [1997]) criteria, where 'essential' information gets two points and 'subsidiary' information gets one point. The Information score is the total number of points thus accumulated. An example would be the utterance: To $\lambda \varepsilon \omega \varphi о \rho \varepsilon i ́ o$ $\varepsilon$ '́vধ. /to leoforio efije/ 'The bus left.' For this production examinees would receive 3 points. They get 1 point for re-introducing the agent (i.e. the bus) and 2 for figuring out its role in the action (i.e. that it left).

B. Subordinates: After each narrative was divided into sentences, the subordinate clauses produced were counted.

C. A5LS: When the narratives were transcribed, they were divided into sentences (T-units). Then the MLU-word was calculated and the mean of the five longest sentences were computed.

D. MLU-word: This was calculated based on words for each narrative (MLUword for all T-units). All words were added up and the sum was divided by sentences produced.

E. Sentences: This measure forms the total number of sentences used (T-unit). 


\section{Results}

Children with SLI were less accurate than age-matched TLD peers on expressive vocabulary measures using the DVIO. The mean scores on the DVIO vocabulary subtest are presented in Table 2 above. For the COAT, the percentages of correct responses were calculated for object and action names as provided by all children and a summary of the results is given in Figure 1 according to picture type.

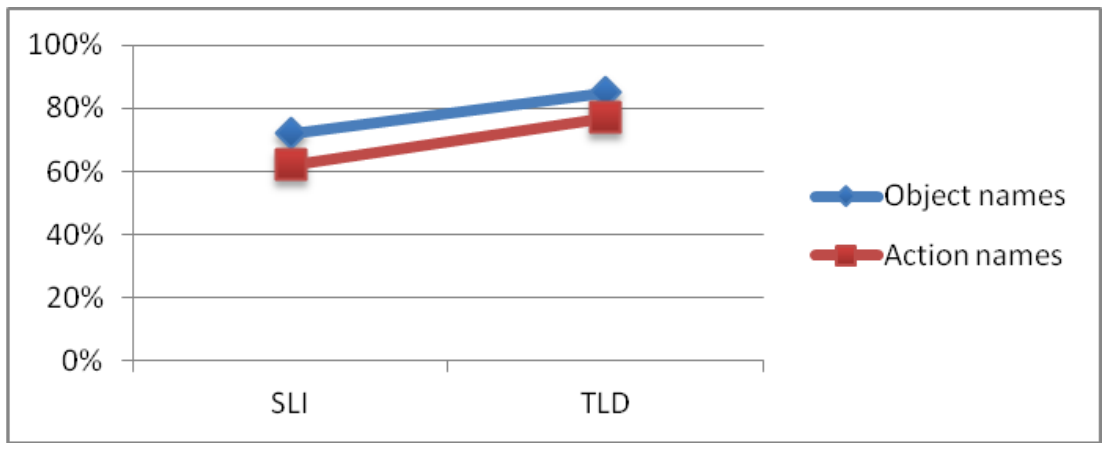

Figure 1. Mean percentages correct for object and action names by both groups.

Independent samples t-tests were performed to test for mean differences between the children with TLD and those with SLI. The latter showed significantly more difficulties retrieving object $\left(t_{19}=-2.735, p=0.013\right)$ and action $\left(t_{19}=-3.940\right.$, $\mathrm{p}=0.001$ ) names compared to TLD peers. For the BST, the mean scores for all five measures are displayed in Table 3. Children with SLI scored significantly worse in three out of the five measures than the children with TLD: Subordinates $\left(t_{19}=-2.815, p=0.011\right)$, A5LS $\left(t_{20}=-2.377, p=0.028\right)$, and MLU-word $\left(t_{20}=-\right.$ $2.942, p=0.008)$. For the other two measures, the mean sores indicated worse performance for the SLI group but the difference failed to reach significance (Information: $t_{20}=-1.663, p=0.112$ and Sentences: $t_{20}=-0.437, p=0.667$ ). Similar results were obtained for all pair-wise comparisons using a non-parametric Mann-Whitney U test.

The scores on the expressive vocabulary subtest of the DVIO and on the COAT were correlated with the number of subordinates, A5LS, and MLU-word based on the BST to determine if expressive vocabulary skills can predict children's narrative performances at the microstructure level. The results are reported in the next section. 
Table 3.

Mean scores for the five measures.

\begin{tabular}{|l|c|c|c|c|}
\hline Child & \multicolumn{2}{|c|}{ TLD (n=11) } & \multicolumn{2}{c|}{ SLI (n=10) } \\
\hline Age & \multicolumn{2}{|c|}{$\mathbf{4 ; 6 - 6 ; 1 1}$} & Mean & St. Dev. \\
\hline & Mean & St. Dev. & 23.5 & 9.419 \\
\hline Inf. Total & 29.8 & 7.264 & 6.4 & 2.047 \\
\hline A5LS & 8.8 & 2.581 & 2.0 & 1.054 \\
\hline Sub. Cl. & 4.1 & 2.071 & 3.8 & 0.685 \\
\hline MLU - words & 5.1 & 1.223 & 17.7 & 3.974 \\
\hline No. of sentences & 18.4 & 3.957 & & \\
\hline
\end{tabular}

\section{Correlations}

The Pearson correlation coefficients between vocabulary measures and variables extracted from the Bus Story (MLU, A5LS, and subordinate clauses) appear in Table 4. The table contains values for the TLD group; correlation coefficients for the SLI group appear in parentheses. There is a positive relationship between the sub-scores of the COAT for both groups (significant only in the TLD group) and a non significant positive trend between the noun component of the COAT and the DVIO-Vocabulary measure. High positive associations exist among the three BST measures; all are significant in the case of the TLD group, but only the A5LS and MLU correlation is significant for the SLI group. Not many of the estimated coefficients turned out to be significant, probably due to the small sample size of the two groups examined. In addition, scores in the SLI sample are lower with a more restricted range than in the TLD sample, hence correlations for the SLI turn out to be even smaller. Overall, no significant relationships were found between vocabulary and BST measures.

Table 4.

Correlation coefficients between the vocabulary and BST measures.

\begin{tabular}{|c|c|c|c|c|c|c|}
\hline & $\begin{array}{c}\text { DVIO } \\
\text { Vocabulary }\end{array}$ & $\begin{array}{l}\text { COAT } \\
\text { Nouns }\end{array}$ & $\begin{array}{l}\text { COAT } \\
\text { Verbs }\end{array}$ & A5LS & $\begin{array}{l}\text { Subordinate } \\
\text { clauses }\end{array}$ & MLU \\
\hline DVIO Vocabulary & & $\begin{array}{c}0.275 \\
(0.388) \\
\end{array}$ & $\begin{array}{c}-0.007 \\
(-0.139)\end{array}$ & $\begin{array}{c}0.098 \\
(-0.458)\end{array}$ & $\begin{array}{c}-0.028 \\
(-0.186)\end{array}$ & $\begin{array}{c}0.178 \\
(-0.549) \\
\end{array}$ \\
\hline COAT Nouns & & & $\begin{array}{c}0.752^{\prime \prime} \\
(0.139) \\
\end{array}$ & $\begin{array}{c}0.335 \\
(-0.540) \\
\end{array}$ & $\begin{array}{c}0.157 \\
(-0.294) \\
\end{array}$ & $\begin{array}{c}0.265 \\
(-0.519) \\
\end{array}$ \\
\hline COAT Verbs & & & & $\begin{array}{c}0.011 \\
(0.200) \\
\end{array}$ & $\begin{array}{l}-0.288 \\
(0.241) \\
\end{array}$ & \begin{tabular}{|l|}
-0.002 \\
$(0.330)$ \\
\end{tabular} \\
\hline A5LS & & & & & $\begin{array}{l}0.767^{\text {sth }} \\
(0.525) \\
\end{array}$ & $\begin{array}{r}0.946^{\text {the }} \\
\left(0.939^{\text {thn }}\right) \\
\end{array}$ \\
\hline Subordinate clauses & & & & & & $\begin{array}{c}0.669 \\
(0.592)\end{array}$ \\
\hline
\end{tabular}

* Correlation is significant at the 0.05 level (2-tailed).

* Correlation is significant at the 0.01 level (2-tailed). 


\section{Discussion}

In the present study, two expressive vocabulary measures (the vocabulary subtest of the DVIO and the action and object naming subtest of the COAT) were correlated with three microstructure measures from the BST (subordinate clauses, A5LS, and MLU-word) in order to determine if single word expressive vocabulary can predict morphosyntactic abilities in school-aged children with TLD and those with SLI. The assumption is that children with larger expressive vocabulary scores would have higher scores on the narrative microstructure measures and children with lower expressive vocabulary skills would show the opposite pattern. Overall, our results showed no significant relation between the Bus Story measures and the expressive vocabulary scores for normal and language-impaired children. It is possible that the limited size of the present sample might have obscured some significant findings.

From our search of the literature, the correlation between expressive vocabulary and morphosyntactic ability as related to narrative retell microstructure has been found significant in some studies but not in others. Specifically, for monolingual English-speaking children, this correlation was reported significant in typically developing 5-year-olds (Adams \& Gathercole 1996) and language-impaired children with a mean age of 8;4 (Howlin \& Kendall 1991). In contrast, studies investigating the relation between the BST measures and vocabulary scores in bilingual populations with TLD report a lack of correlation (Schelletter \& Parke 2004, Simon-Cereijido \& Guitierrez-Clellen 2009). (Expressive vocabulary was measured using the Word Finding Vocabulary Test for English (Renfrew 1995).) Both groups of researchers claim that vocabulary skills in bilingual children do not predict syntactic ability (at least in typical development). It thus appears that our results are comparable with studies from the bilingual literature.

This perhaps unexpected finding raises the interesting topic of linguality in Greek Cypriot children. As mentioned above, Cypriot Greek does differ from Standard Modern Greek in many ways, which go beyond the sound system and lexical inventory. With clear morphosyntactic differences, such as clitic placement (Terzi 1999 and subsequent work), wh-question formation (e.g. Grohmann et al. 2006), and a host of other structural properties of the grammar (Tsiplakou et al. forthcoming), one possible conclusion might be that the two varieties are so much different as to allow classification as two separate languages. In that most extreme case, Greek Cypriots could then be characterized as bilingual speakers with Cypriot Greek as their native first language and Standard Modern Greek as possibly simultaneous bilingual acquisition or early child second language acquisition. If that were the case, the results from the present study would be in line with similar findings from bilingual acquisition cited above.

However, we are not yet at a point at which we can unequivocally sustain such a stance. After all, Cypriot and Standard Modern Greek are also very close 
to one another. In addition, qualitatively the standard variety spoken in Cyprus differs distinctly from that spoken in mainland Greece, for example. Lastly, in the context of diglossia, the distinction between the two varieties, for all practical and theoretical purposes, seems to lie in the sociolinguistic domain more so than in the formal grammatical domain, also for political reasons. We will not review the continuously growing body of work here but confine ourselves to a simple point: With no native speakers of Standard Modern Greek in the Greek Cypriot population, the bilingual classification in the traditional sense faces significant obstacles. Likewise, if the sociolinguistic situation is indeed that of diglossia, the "high" variety can hardly be a separate language (again, without changing the respective definitions accordingly). We therefore work on the assumption that in terms of linguality, Greek Cypriot speakers are "bi- $x$ ", where ' $x$ ' needs to be further determined, possibly beyond 'varietal', 'lingual', 'dialectal', etc. (Grohmann 2011, Grohmann \& Leivada 2012). One possible value for $x$ could be 'lectal' (Rowe $\&$ Grohmann 2013): Greek Cypriots are bilectal speakers of (at least) two Greek lects.

We hope to flesh out this idea in the near future. Whatever bi-x may turn out to be, it looks, however, as if bi-xsuch as bilectal speakers behave, with respect to the task at hand, more like bilingual than monolingual speakers. That is to say that competence in another lect may indeed contribute to (meta)linguistic abilities that go beyond monolingual speakers. In order to support such an approach, similar studies could be carried out in less strong settings than Cyprus, perhaps with Greek speakers from the islands such as the Dodecanese (which shows similarities to the grammar of Cypriot Greek) or Crete and compare them with mainland Greek speakers or investigate urban versus rural settings in Greece - or other countries for other languages with significant and less significant dialectal variation (German, French, English, etc.). This project is clearly one for the future, hence our conclusion at this point can only be tentative: Greek Cypriot children pattern more with bilinguals than monolinguals with respect to a possible correlation between expressive vocabulary and morphosyntactic ability as related to narrative retell microstructure. 


\section{References}

Adams, A.-M. and S.E. Gathercole. 1996. Phonological working memory and spoken language development in young children. Quarterly Journal of Experimental Psychology Section A: Human Experimental Psychology 49(1), 216233.

Dunn, L. and L. Dunn. 1981. Peabody Picture Vocabulary Test. Circle Pines, MN: American Guidance Service.

Gillam, R.B. and N. Pearson. 2004. Test of Narrative Language. Austin, TX: Pro-Ed.

Grohmann, K.K. 2011. Some directions for the systematic investigation of the acquisition of Cypriot Greek: A new perspective on production abilities from object clitic placement. In E. Rinke and T. Kupisch (eds.), The Development of Grammar: Language Acquisition and Diachronic Change. Amsterdam: John Benjamins, 179-203.

Grohmann, K.K. and E. Leivada. 2012. Interface ingredients of dialect design: Bi-X, socio-syntax of development, and the grammar of Cypriot Greek. In A.M. Di Sciullo (ed.), Towards a Biolinguistic Understanding of Grammar: Essays on Interfaces. Amsterdam: John Benjamins, 239-262.

Grohmann, K.K., P. Panagiotidis and S. Tsiplakou. 2006. Properties of wh-question formation in Cypriot Greek. In M. Janse, B.D. Joseph and A. Ralli (eds.), Proceedings of the 2nd International Conference on Modern Greek Dialects and Linguistic Theory (Mytilene, Greece: 30 September-3 October 2004). Patras: University of Patras, 83-98.

Grohmann, K.K., E. Theodorou, N. Pavlou, E. Leivada, E. Papadopoulou and S. Martínez-Ferreiro. 2012. The development of object clitic placement in Cypriot Greek and the Romance connection. In S. Ferré, P. Prévost, L. Tuller and R. Zebib 
(eds.), Selected Proceedings of the Romance Turn IV Workshop on the Acquisition of Romance Languages. Newcastle-upon-Tyne: Cambridge Scholars Publishing, 128-152.

Howlin, P. and L. Kendall. 1991. Assessing children with language tests — which tests to use? British Journal of Disorders of Communication 26, 355-367.

International Phonetic Association. 1999. Handbook of the International Phonetic Association: A Guide to the Use of the International Phonetic Alphabet. Cambridge: Cambridge University Press.

Kambanaros, M. and K.K. Grohmann. 2010. Patterns of object and action naming in Cypriot Greek children with SLI and WFDs. In K. Franich, L. Keil, K. Iserman and J. Chandlee (eds.), Proceedings of the 34th Boston University Child Language Development-Supplement.

Kambanaros, M., K.K. Grohmann and M. Michaelides. 2013. Lexical retrieval for nouns and verbs in typically developing bilectal children. First Language 33, 182-199.

Leonard, L. 1998. Children with Specific Language Impairment. Cambridge, MA: MIT Press.

Manhardt, J. and L. Rescorla. 2002. Oral narrative skills of late talkers at ages 8 and 9. Applied Psycholinguistics 23, 1-21.

Newman, R.S. and D.J. German. 2002. Effects of lexical factors on lexical access among typical language-learning children and children with word-finding difficulties. Language and Speech 45, 285-317.

Raven, J., J.C. Raven and J.H. Court. 2000. Manual for Raven's Progressive Matrices and Vocabulary Scales. San Antonio, TX: Harcourt Assessment.

Renfrew, C. 1969 [1997]. The Bus Story Test: A Test of Continuous Speech, $4^{\text {th }}$ ed. Oxford: North Place, Old Headington.

Renfrew, C. 1995. Word Finding Vocabulary Test. Bicester: Winslow.

Rice, M.L. 2003. A unified model of specific and general language delay: grammatical tense as a clinical marker of unexpected variation. In Y. Levy and ]. Schaeffer (eds.), Language Competence across Populations: Toward a Definition of Specific Language Impairment. Mahwah, N]: Lawrence Erlbaum, 63-94. 
Rowe, C. and K.K. Grohmann. 2013. Discrete bilectalism: Towards co-overt prestige and diglossic shift in Cyprus. International Journal of the Sociology of Language 224: 119-142.

Schelletter, C. and T. Parke. 2004. Using story re-tell in bilingual assessment. Academic Exchange Quarterly 8, 158-162.

Simon-Cereijido, G. and V.F. Gutiérrez-Clellen. 2009, A cross-linguistic and bilingual evaluation of the interdependence between lexical and grammatical domains. Applied Psycholinguistics 30, 315-338.

Stavrakaki, S. and I.M. Tsimpli. 2000. Diagnostic verbal IO test for Greek preschool and school age children: Standardization, statistical analysis, psychometric properties [in Greek]. Proceedings of the 8th Conference on Speech Therapy. Athens: Ellinika Grammata, 95-106.

Terzi, A. 1999. Cypriot Greek clitics and their positioning restrictions. In A. Alexiadou, G. Horrocks and M. Stavrou (eds.), Studies in Greek Syntax. Dordrecht: Kluwer, 227-240.

Theodorou, E. 2012. Narratives in Cypriot Greek children with SLI. In K.K. Grohmann, E. Shelkovaya and D. Zoumbalides (eds.), Linguists of Tomorrow: Selected Papers from the 1st Cyprus Postgraduate Conference in Theoretical and Applied Linguistics. Newcastle-upon-Tyne: Cambridge Scholars Publishing, 205231.

Theodorou, E. 2013. Diagnosing specific language impairment: The case of Cypriot Greek. PhD thesis, University of Cyprus.

Theodorou, E. and K.K. Grohmann. 2010. Narratives in Cypriot Greek monoand bilingual children with SLI. In A. Botinis (ed.), Proceedings of ISCA Tutorial and Research Workshop on Experimental Linguistics 2010 - 25-27 August 2010, Athens, Greece. Athens: ISCA and the University of Athens, 185-188.

Tsiplakou, S., P. Coutsougera, and P. Pavlou. Forthcoming. A Contemporary Grammar of Cypriot Greek. Munich: Lincom Europa.

Vogindroukas, I., A. Protopappas, and G. Sideris. 2009. Word-Finding Test. Chania: Glafki. 
Major Trends in Theoretical and Applied Linguistics 